\title{
Sequence determinants of in cell condensate assembly morphology, dynamics, and oligomerization as measured by number and brightness analysis
}

Ryan J. Emenecker ${ }^{1,2,3}$, Alex S. Holehouse ${ }^{1,2 *}$, Lucia C. Strader ${ }^{2,3,4 *}$

1. Department of Biochemistry and Molecular Biophysics, Washington University School of Medicine, St. Louis, MO 63110, USA

2. Center for Science and Engineering Living Systems (CSELS), Washington University, St. Louis, MO 63130, USA

3. Center for Engineering Mechanobiology, Washington University, St. Louis, MO 63130, USA

4. Dept. of Biology, Duke University, Durham, NC 27708

*Correspondence: lucia.strader@duke.edu, alex.holehouse@wustl.edu 


\begin{abstract}
Background: Biomolecular condensates are non-stoichiometric assemblies that are characterized by their capacity to spatially concentrate biomolecules and play a key role in cellular organization. Proteins that drive the formation of biomolecular condensates frequently contain oligomerization domains and intrinsically disordered regions (IDRs), both of which can contribute multivalent interactions that drive higher-order assembly. Our understanding of the relative and temporal contribution of oligomerization domains and IDRs to the material properties of in vivo biomolecular condensates is limited. Similarly, the spatial and temporal dependence of protein oligomeric state inside condensates has been largely unexplored in vivo. Methods: In this study, we combined quantitative microscopy with number and brightness analysis to investigate the aging, material properties, and protein oligomeric state of biomolecular condensates in vivo. Our work is focused on condensates formed by AUXIN RESPONSE FACTOR 19 (ARF19), which is a transcription factor integral to the signaling pathway for the plant hormone auxin. ARF19 contains a large central glutamine-rich IDR and a C-terminal Phox Bem1 (PB1) oligomerization domain and forms cytoplasmic condensates.

Results: Our results reveal that the IDR amino acid composition can influence the morphology and material properties of ARF19 condensates. In contrast the distribution of oligomeric species within condensates appears insensitive to the IDR composition. In addition, we identified a relationship between the abundance of higher- and lower-order oligomers within individual condensates and their apparent fluidity.

Conclusions: IDR amino acid composition affects condensate morphology and material properties. In ARF condensates, altering the amino acid composition of the IDR did not greatly affect the oligomeric state of proteins within the condensate.
\end{abstract}




\section{Background}

Across all kingdoms of life, cells must accomplish the difficult task of organizing their intracellular environment. Cells can accomplish this through two primary mechanisms; compartmentalization through use of membrane-bound organelles or through the formation of biomolecular condensates [1-4]. Biomolecular condensates are non-stoichiometric assemblies of biomolecules that are defined by their common feature of spatially concentrating cellular components [3]. Growing evidence suggests that in many cases, biomolecular condensates form through the process of phase separation [4-8]. Of fundamental importance to this process is the multivalency of the molecules that undergo phase separation [8-10]. Multivalency refers to the capacity of a molecule to simultaneously engage in multiple intermolecular interactions. The ability of multivalent biomolecules to undergo coordinated and regulatable assembly is at the heart of biomolecular condensate formation, regardless of if the underlying mechanism is phase separation or some other process.

Many proteins that are capable of undergoing phase separation contain intrinsically disordered regions (IDRs) [7,11-14]. IDRs are protein regions that do not adopt a fixed three-dimensional structure but instead exist as an ensemble of conformations that interconvert between one another [15-17]. While IDRs are not strictly necessary for phase separation, in a number of specific biological systems IDRs have been found to be necessary and/or sufficient for phase separation and condensate formation [12,18-21]. However, folded domains often play key roles in facilitating initial oligomerization that licenses subsequent phase separation and can in their own right undergo phase separation absent IDRs [10,22-34]. Taken together, it should be clear that the molecular basis for multivalency is not constrained to a specific class of biomolecule.

The observation that IDRs can drive phase separation is often taken as evidence that they have evolved to facilitate biomolecular condensate formation. An alternative interpretation is that even if condensate formation is driven by folded domains, multivalent IDRs may be essential for the formation of dynamic, labile, and functionally responsive biomolecular assemblies $[35,36]$. Under this model, IDRs offer a means to encode locally tethered molecular lubricants that prevent aberrant assembly of folded domains and to tune the material state of biomolecular condensates [20,27,36-39]. With this in mind, understanding how structurally or chemically orthogonal multivalent interactions can tune condensate material properties represents an emerging set of questions.

Condensates are frequently well-described as viscoelastic materials, meaning they have an elastic response upon deformation and will also drip, flow, or wet like a viscous fluid [6]. The apparent viscosity of a condensate can range from liquids that rearrange in milliseconds to viscous solid-like assemblies that fuse on the order of hours or longer [40,41]. A growing body of literature supports an emerging view that condensate material state can be a key determinant of biological function [38-40,42-44]. With this in mind, a molecular understanding of interactions that determine material state represents an important next step in our ultimate goal of relating protein sequence and structure to cellular function. The viscosity of a condensate depends on 
the lifetime and the number of cohesive multivalent interactions that are responsible for assembly $[7,9,45]$. Previous work focused on IDRs has shown that amino acid composition and patterning can influence condensate dynamics in a manner that alters these parameters [20,46-48]. However, many phase separating proteins possess a modular protein architecture that include (at a minimum) an oligomerization domain and an IDR [10]. For these modular proteins, the relative impact of folded vs. disordered domains on the emergent material properties of a condensate is less well studied.

A final confounding factor when considering how IDRs tune the material properties of condensates emerges from the observation that IDRs are inherently sensitive to their solution environment [49-54]. Given the crowded and complex milieu of macromolecules, osmolytes, and ions in vivo, one may expect that the material properties for a condensate measured in vitro to be substantially different, an expectation supported by numerous studies. As such, to understand physiologically relevant determinants of condensate material state, ideally measurements of condensate dynamics and protein oligomeric state would be made in live cells.

Here we leverage the previously characterized modular transcription factor AUXIN RESPONSE FACTOR 19 (ARF19) from Arabidopsis thaliana as a model system to probe the determinants of condensate properties and protein oligomeric state in cells. Auxin is a plant hormone that is involved in essentially all plant growth and developmental processes [55]. ARF19 condensate formation has been proposed to be a mechanism by which signaling through the auxin signaling pathway is attenuated [22]. Notably, disruption of ARF19 condensate formation has a dramatic impact on the expression of auxin-responsive genes, implicating ARF19 condensates as global remodellers of auxin-dependent transcription [22]. ARF19 is composed of a DNA-binding domain followed by a large glutamine-rich IDR and a C-terminal Phox Bem1 (PB1) oligomerization domain. Importantly, in vivo, we have previously shown that the oligomerization domain and the IDR are essential for condensate formation [22]. As such, ARF19 is a convenient model system to examine the contribution of the IDR to material properties of condensates formed by proteins containing both IDRs and oligomerization domains. 


\section{Methods}

\section{Plant Growth}

Seeds were surface sterilized [56] and then suspended in $0.1 \%$ agar. Suspended seeds were then kept at $4^{\circ} \mathrm{C}$ for 2 days for stratification. After stratification, seeds were plated on plant nutrient $(\mathrm{PN})$ medium [57] $+0.5 \%(\mathrm{w} / \mathrm{v})$ sucrose solidified with $0.6 \%$ agar. Seeds were then grown for 1 week at $22^{\circ} \mathrm{C}$ under continuous light before being transplanted to soil. Once transplanted to soil, seedlings were grown under long day (16 hours light : 8 hours of dark) conditions for 2-3 more weeks before being used for protoplast generation.

\section{Protoplast Isolation}

For all protoplast transfections, an arf19, arf7 double mutant in the Col-0 background was used to minimize the risk of native ARF19 or ARF7 interactions impacting condensate formation. Protoplasts were isolated from 3-4 week-old-plants via the tape-method as described in [58] with slight modifications. Briefly, after the upper epidermal surfaces of the leaves were peeled, peeled leaves were incubated in enzyme solution ( $1 \%$ cellulase, $0.25 \%$ macerozyme, $0.4 \mathrm{M}$ mannitol, $20 \mathrm{mM} \mathrm{KCl}, 20 \mathrm{mM} \mathrm{MES}, 10 \mathrm{mM} \mathrm{CaCl}_{2}, 0.1 \% \mathrm{BSA}$ ) in 6-well plates as opposed to Petri dishes. In addition, peeled leaves were incubated with the enzyme solution for 60-90 minutes. Lastly, leaves were shaken at 60 RPM during incubation with the enzyme solution.

\section{Protoplast Transfection}

Protoplast transfection followed methods as described in [58] with slight modifications. After resuspension in the $\mathrm{MMg}$ solution, $150 \mu \mathrm{l}$ of protoplasts were mixed with 10-12 $\mathrm{gg}$ of plasmid DNA. Next, protoplasts were mixed with an equal volume of the PEG solution and rocked back and forth gently to mix the protoplasts with the PEG solution. Once mixed, protoplasts were incubated for 10-12 minutes before $660 \mu$ of W5 solution was added. After protoplast transfection, protoplasts were immediately suspended in $2 \mathrm{ml}$ of $\mathrm{W} 1$ buffer [58] and then dispensed onto $50 \times 7 \mathrm{~mm}$ round bottom glass dishes (Ted Pella Inc, product number 14035-120). All expressions in protoplasts utilized the UBQ10 promoter, and ARF19 as well as the two ARF19 variants contained an $\mathrm{N}$-terminal mVenus tag.

\section{Vector Construction}

The transient protoplast expression vectors for ARF19 and ARF19 QtoS were made through recombination from a pENTR vector into pUBQ10:mVenus-GW and have been described previously [22]. For the ARF19 QtoG variant, the ARF19 QtoG IDR was synthesized with 8 base pairs of overhangs to the region immediately upstream of the ARF19 IDR at the $5^{\prime}$ end and 20 base pairs of overhang to the region immediately downstream of the ARF19 IDR at the 3' end as a gBlock by Integrated DNA Technologies. Then a pENTR-ARF19 vector was linearized via PCR amplification using Pfx Platinum polymerase (Life Technologies) such that it no longer contained the wild-type IDR, and a 12 base pair overhang with the 5 ' end of the QtoG IDR 
gBlock was added at this step using the primers 5'-AACTAGACTTAAACCAGGGAACATC-3' and 5'-AACTTGGTTCCCAACTATGGC-3'. The resultant PCR product and the QtoG IDR gBlock were used in an In-Fusion cloning reaction (Takara) to generate pENTR-ARF19 QtoG IDR. After sequence confirmation, pENTR-ARF19 QtoG IDR was recombined into the pUBQ10:mVenus-GW vector using LR Clonase II (ThermoFisher) to generate pUBQ10:mVenus-ARF19 QtoG, which was subsequently sequence confirmed.

\section{Microscopy Imaging}

Immediately after transfection, protoplasts suspended in $2 \mathrm{ml}$ of $\mathrm{W} 1$ were dispensed onto Ted Pella Inc 50x7 mm PELCO Round Bottom Dishes (glass, 40 mm) (product number 14035-120). The protoplasts were then incubated for approximately 16 hours in the round bottom dish with a vacuum grease sealed lid enclosing the dish such that the dish did not dry out and alter the concentrations of solutes prior imaging. After approximately 16 hours, the lid was removed from the dish and the protoplasts were placed on the confocal stage for imaging. Imaging was carried out using the Leica SP8 confocal microscope. All images of condensates used the HyD detector and a 40x water immersion lens. All images in Figure 1C used the Leica Lightning Imaging module and Lightning deconvolution. For the time lapses of condensate fusion events in figure $4 \mathrm{~A}$, images were obtained from time lapses of individual whole protoplasts. Unlike the images presented in Figure 1C, the images in figure 4A did not use the Leica Lightning module and used the 20x, dry-immersion objective.

\section{Number and Brightness Imaging and Analysis}

All imaging for N\&B was taken on a Leica SP8 using a HyD detector in photon counting mode using a 40x water immersion objective. Prior to imaging, slight adjustments to the correction collar on the objective were made as needed to minimize differences in acquired signal due to differences in glass thickness of the round bottom dishes. The laser used was a $514 \mathrm{~nm}$ set at $0.01 \%$ power, and the range of wavelengths used for capturing the images were those between $519 \mathrm{~nm}$ and $550 \mathrm{~nm}$. The pinhole was set at $1 \mathrm{AU}$, the scan speed was $310 \mathrm{~Hz}$, and the zoom was set at 11.5. One hundred consecutive frames were captured for each data point, and the interval between each frame was 0.839 seconds ( 1 minute, 23.871 seconds total acquisition time). Pixel dwell time was $8.19 \mu \mathrm{sec}$. Images were captured at a $256 \times 256$ format. For analysis, we used the SimFCS software [59]. We used protoplasts expressing mVenus under the UBQ10 promoter in order to calibrate software parameters such as the S-factor. Specifically, the region that quantified monomer brightness was determined using protoplasts expressing mVenus by selecting the fluorescence-positive pixels with a cursor. Using the assumption that anything twice the brightness of the calibrated monomer would be a dimer, three times the brightness would be a trimer, and so forth, the amount of higher order oligomers was quantified. For our analyses, monomers, dimers, 3-10mers, and 10+-mers were quantified with the exception of Figure 5A. For Figure 5A, analysis of the different oligomeric species in the condensates was carried out similar to as before with the exception that individual species from monomers to decamers and 10+-mers were individually analyzed in order to obtain images 
showing their distribution within each condensate. Then, the images were uploaded to Adobe Illustrator and overlaid with one another. Finally, each pixel was false colored such that each pixel corresponded to the oligomeric species identified in the N\&B analysis.

\section{FRAP Imaging and Analysis}

FRAP imaging was carried out on a Leica SP8 using a PMT detector and a 40x water immersion objective using the Leica FRAP module. All FRAP imaging was carried out immediately after N\&B imaging. Pre- and post-photobleaching image acquisition used a $514 \mathrm{~nm}$ laser at $0.06 \%$ power with a range of acquisition of $519 \mathrm{~nm}$ to $550 \mathrm{~nm}$. All imaging used a $512 \times 512$ format and a scan speed of $1400 \mathrm{hz}$. One pre-bleach image was acquired followed by the photobleaching and then 120 post-bleach images were captured at 1 second intervals. The duration per acquisition of each image was 0.371 seconds. For FRAP imaging, the zoom was adjusted as needed depending on the size of the condensate. All optional SP8-specific FRAP module settings were set as follows: fly mode - off, zoom in - on, change bleach format - off, set background to zero - off, delete bleach images after scan - off. For photobleaching, the $448 \mathrm{~nm}$, $488 \mathrm{~nm}, 514 \mathrm{~nm}$, and $552 \mathrm{~nm}$ lasers were set to 100\% power and targeted to approximately one half of the condensate for a total of 1.8762 seconds. Following image acquisition, data was imported into FIJI (FIJI Is Just ImageJ) [60] in the original .lif file format for analysis. The percent recovery was determined by quantifying the amount of recovery observed in the photobleached region post-photobleaching. 


\section{Results}

\section{Altering IDR composition dramatically impacts intracellular condensate morphology and dynamics}

ARF19 contains a $~ 500$-residue glutamine-rich IDR that includes a large prion-like domain (PLD), a class of low-complexity IDR enriched in polar amino acids [61,62]. The ARF19 IDR lies between an N-terminal DNA binding domain and a C-terminal PB1 oligomerization domain (Figure 1A). Given its large size and strong sequence bias, we wondered if changing the amino acid composition (while maintaining the natural enrichment for polar amino acids) would alter condensate properties.

To explore how IDR composition impacts condensates formed by ARF19 in vivo, we took advantage of a transient expression protoplast system. Protoplasts are individual spherical cells in which the cell wall has been removed through enzymatic degradation. For our studies, we used protoplasts derived from leaf mesophyll cells isolated from three-week-old $A$. thaliana. Importantly, using protoplasts allowed us to examine the behavior of the ARF19 condensates within the cellular environment. In addition to offering an in vivo environment, protoplasts provide a convenient system to examine condensates for several reasons. The large size of protoplasts makes it easy to image the condensates, their ease of transfection makes it easy to examine many different condensate-forming proteins, and the ability to detect protein expression after transfection in as little as 90 minutes allows one to quickly examine the events leading up to condensate formation. In addition, due to the tight temporal control that transient expression affords, it is possible to estimate condensate age. This enables us to examine condensate formation at a single time point after transfection, an important feature given some condensate properties can change dramatically in a time-dependent manner.

We examined the morphology of condensates in protoplasts formed by full-length ARF19 with a wild-type IDR, an IDR where all glutamines were changed to glycines (QtoG) or an IDR where all glutamines were changed to serines (QtoS) (Figure 1B). Glycine and serine were chosen here as amino acids frequently found in condensate-forming IDRs that retain the polar chemistry of glutamine sidechains yet alter steric and physicochemical properties [21]. Condensates formed by our three constructs displayed striking differences in morphology (Figure 1C). Condensates formed by the WT IDR were in general large, amorphous multilobed assemblies in line with our previous work [22]. In contrast, QtoS condensates were smaller and more spherical, whereas QtoG condensates were intermediate in terms of morphology. Given the oligomerization domain is necessary for condensate formation in plants when expressed at physiological levels, these results reveal that condensate formation and condensate morphology can be uncoupled from one another [22]. 
A.

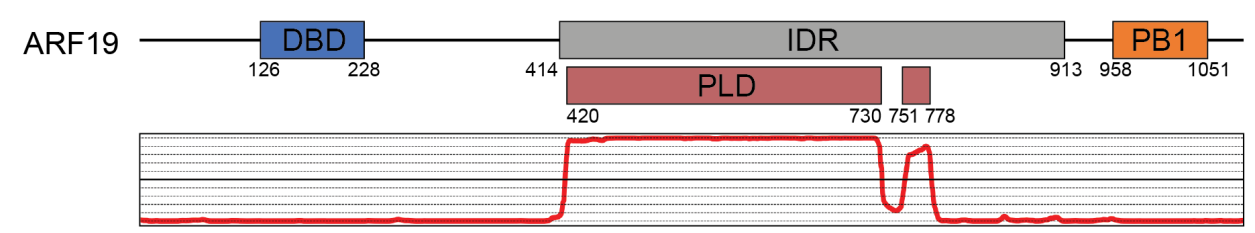

B.

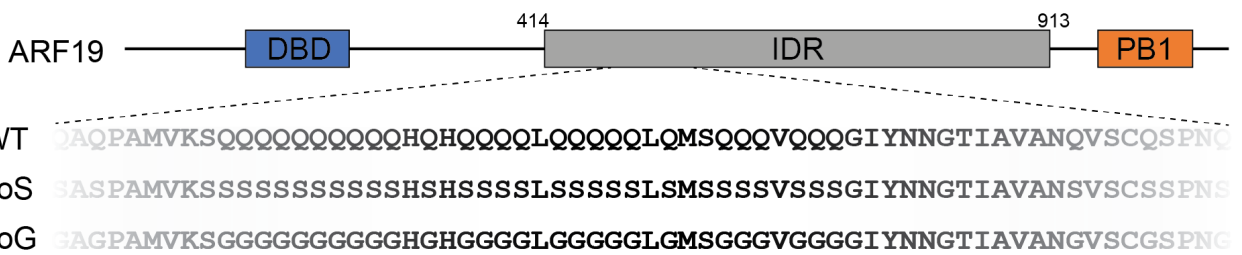

C.
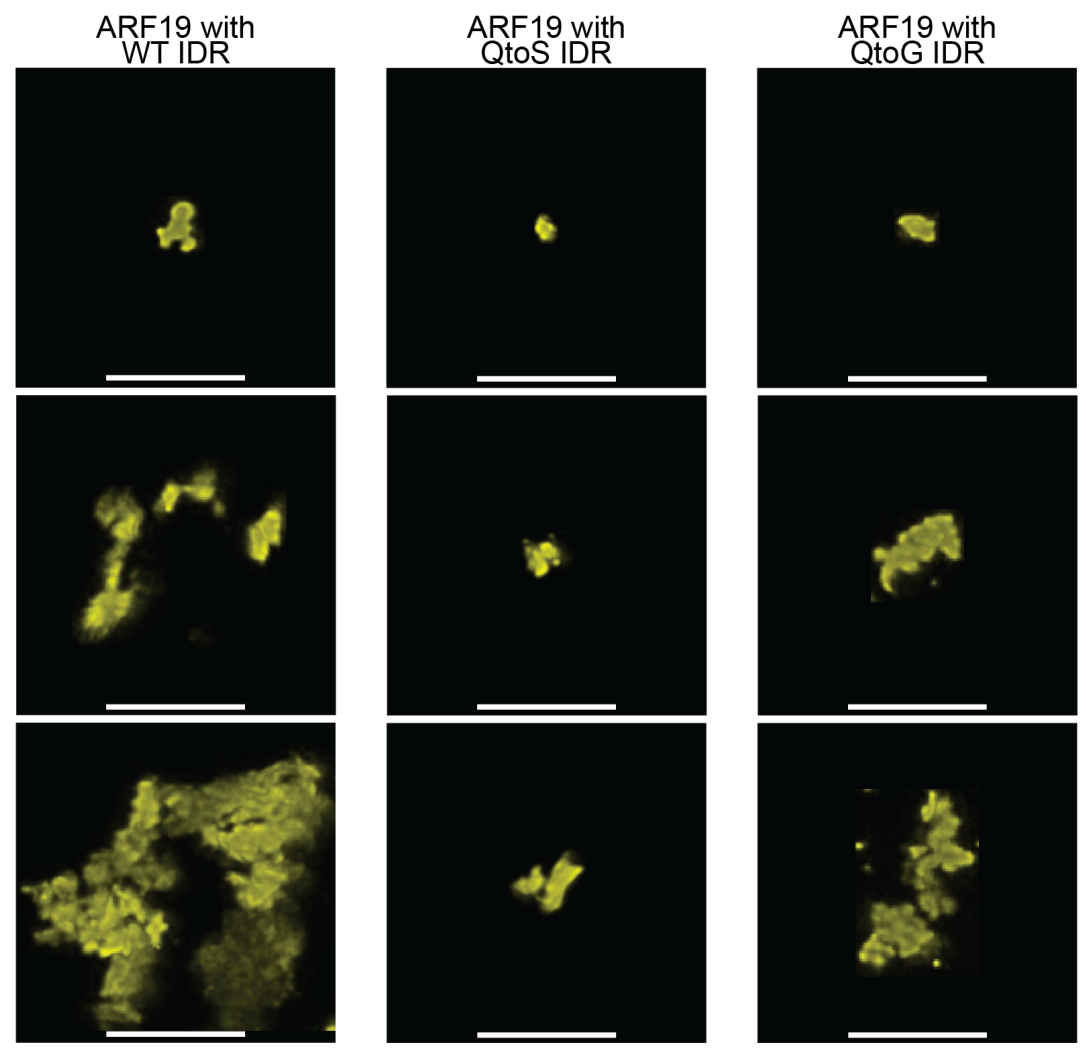

Figure 1. Altering composition of the ARF19 IDR impacts the morphology of ARF19 condensates. (A) Schematic of the ARF19 protein showing the location of the DBD and PB1 domains. The graph below shows predicted prion-like domains within the ARF19 IDR, which were predicted using PLAAC [61]. (B) Schematic showing a subsection of the ARF19 IDR highlighting differences in IDR composition for the QtoS (middle) or QtoG (bottom) variants. While this schematic only shows a subsection of the IDR, for the QtoG or QtoS variants, all glutamines were changed to glycine or serine, respectively. (C) Images showing the range of condensate morphologies formed by wild-type ARF19 or ARF19 with the altered IDR compositions. Images were chosen to represent the breadth of condensate morphology and sizes that are frequently observed across protoplasts when expressing the various constructs. All images were taken in different protoplasts approximately 16 hours after transfection. Scale bars represent 5 microns. 
Condensate morphology is inherently linked to condensate dynamics. For condensates with liquid-like properties, condensate morphology favors spherical assemblies that minimize the interface between the dense and dilute phases. In contrast, for solid-like condensates, morphology is dictated by intramolecular interactions that are inherently anisotropic, giving rise to amorphous assemblies, networked solids, sheets, cross-linked polymers, or any number of non-spherical assemblies [14,25,39,63-66]. However, spherical condensates that rapidly mature from liquid to solids have been observed in many systems, with a 'bunch of grapes' type architecture typifying systems in which arrested spherical assemblies adsorb onto one another $[27,67]$. As such, we suspected that the dynamics of the three variants were likely impacted by the differing IDR compositions. For example, the more spherical morphology of the QtoS condensates may imply enhanced dynamics compared to the more irregular WT condensates.

To assess this, we examined the dynamics of the different ARF19 variants using half-condensate Fluorescence Recovery After Photobleaching (FRAP). Importantly, because in many cases condensate undergo a time-dependent loss in dynamics [41,44], all FRAP measurements were carried out approximately sixteen hours after protoplast transfection.

Condensates formed from all three IDR variants showed relatively low levels of fluorescence recovery. Consistent with prior in planta, wild-type ARF19 condensates exhibited minimal recovery after photobleaching in protoplasts with an average percentage recovery of just $\sim 7 \%$ after two minutes (Figure 2). In contrast, we found that the QtoS and QtoG IDR variants exhibited slightly more liquid-like properties, both having $\sim 9.6 \%$ recovery after two minutes. Despite previous examples where changing glutamine to glycine resulted in more liquid-like condensate dynamics, we found that the QtoG variant was only slightly more liquid-like than wild-type (Figure 2A, 2B) [20]. Nonetheless, this demonstrates that the IDR can influence the morphology and dynamics of ARF19 condensates despite the essential role of the oligomerization domain in their assembly. 


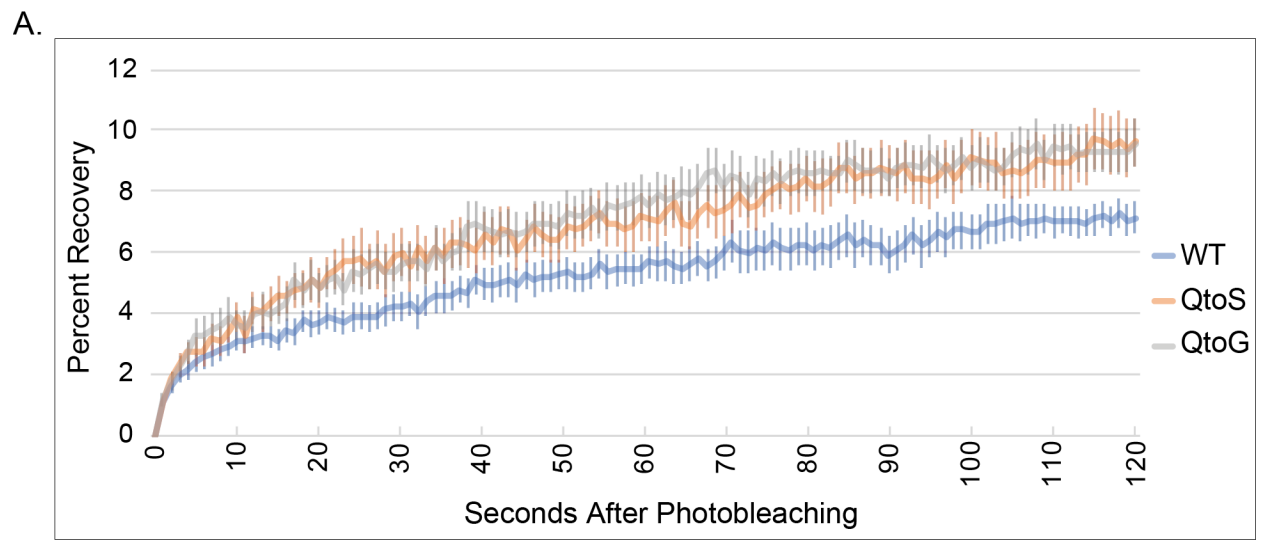

B.

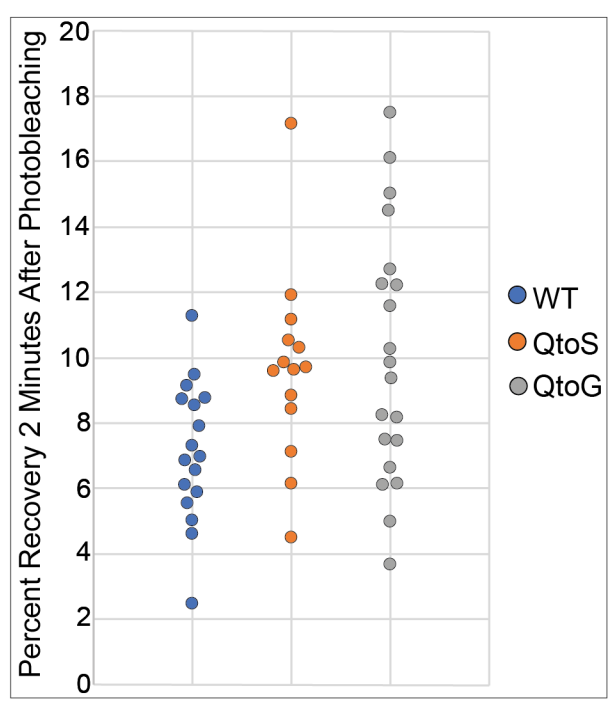

C.

\begin{tabular}{|c|c|c|c|}
\hline & WT & QtoS & QtoG \\
\hline Average \% Recovery & 7.16 & 9.63 & 9.58 \\
\hline Standard Deviation & 2.11 & 2.94 & 3.44 \\
\hline P-value (var. to WT) & N/A & 0.01 & 0.009 \\
\hline
\end{tabular}

Figure 2. IDR composition impacts the fluidity of ARF19 condensates. (A) Average fluorescence recovery curves for the three ARF19 variants. Error bars report standard error of the mean. (B) Each point represents the percent recovery 2 minutes post-photobleaching of an individual condensate. For all FRAP experiments, only one half of the condensate was photobleached. $N=17$ (WT), $N=14$ (QtoS), $N=20$ (QtoG). (C) A table summarizing data from panel A. P-values were calculated using a two-sided t-test comparing the values from wild-type to each of the two IDR variants.

\section{Number and Brightness analysis reveals oligomeric state of ARF19 condensates}

As a means of interrogating the impact that IDR composition has on the oligomeric state of the ARF19 condensates, we utilized number and brightness (N\&B) analysis. N\&B is a fluorescence microscopy method that uses a series of images taken over time to measure the average number of molecules and their oligomeric state in each pixel [68]. In this context, the term oligomeric state describes whether a given molecule behaves as a single unit (i.e. a monomer) or as a higher order assembly (i.e., dimers, trimers etc.).

As with the FRAP measurements, the N\&B measurements were taken approximately sixteen hours after protoplast transfection. For each condensate, we quantified the percentage 
of monomers and dimers (termed 'lower order' oligomers) and the percentage of oligomers equal to ten or higher (termed 'higher order' oligomers).

To our surprise, regardless of whether the percentage of monomers and dimers or the percentage of 10+-mers were examined, there was very little difference in the average oligomeric state between the three variants (Figure $3 \mathrm{~A}, 3 \mathrm{~B}, 3 \mathrm{C}$ ). This is in contrast to our FRAP measurements where we saw an increase in the average fluorescence recovery of condensates formed by the QtoS and QtoG variants. These results support a model whereby IDR composition has the capacity to influence the morphology and dynamics of the ARF19 condensates but not the oligomeric state.

A.

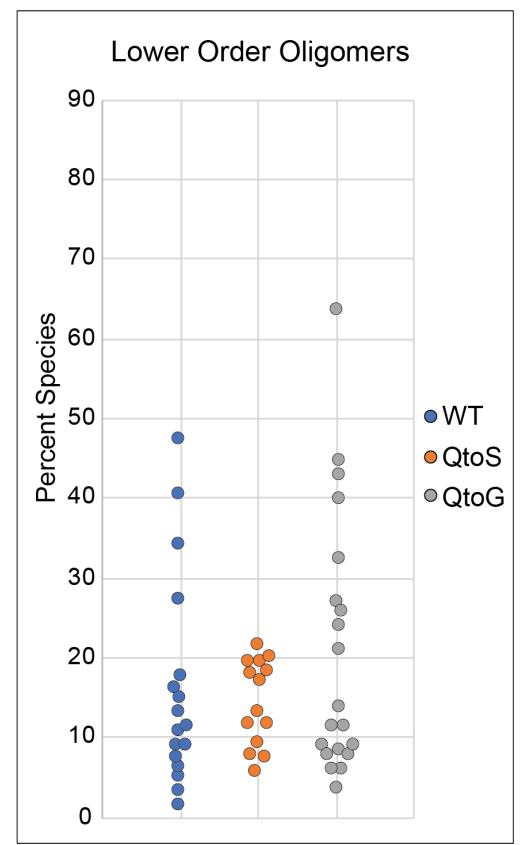

C.

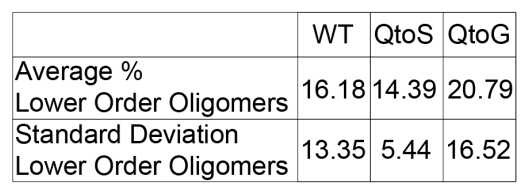

B.

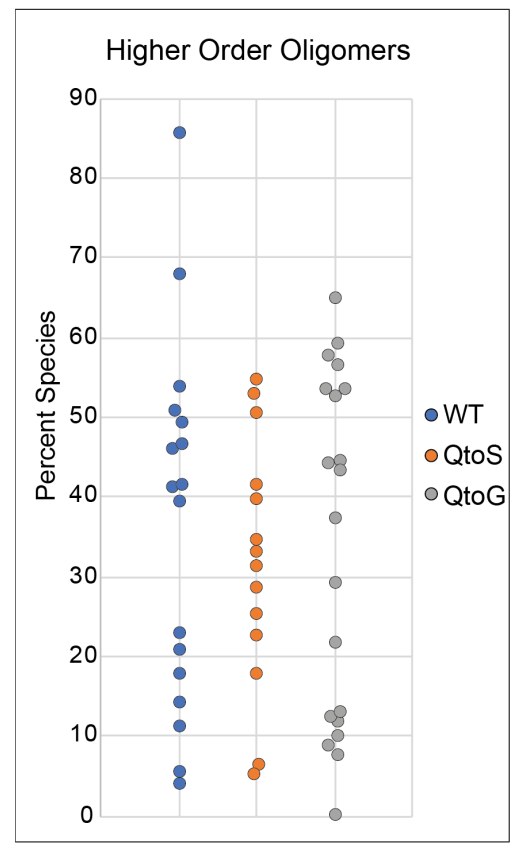

D.

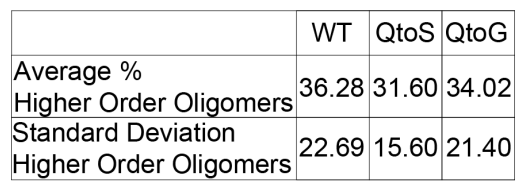

Figure 3. IDR composition has little impact on the oligomeric state of ARF19 condensates. (A) Each point represents the percent of monomers and dimers out of the total number of measured oligomers for an individual condensate. (B) Each point represents the percent of 10+-mers out of the total number of measured oligomers for an individual condensate. (C) A table summarizing the data from figure panel A. (D) A table summarizing the data from figure panel B. Statistical testing is not shown because none of the comparisons were statistically significant. 
Biomolecular condensates frequently undergo a time-dependent maturation in their material state. Condensates often form with liquid-like properties but may undergo a persistent loss of dynamics over time in a process referred to as aging, maturation, or as a liquid-to-solid transition [41,44,69,70]. In investigating the relationship between oligomeric state and condensate dynamics in ARF19, we found that the ARF19 condensates also exhibit a decrease in apparent fluidity over time. Time lapses of protoplasts immediately after transfection show that the condensates initially readily fuse with one another suggesting they are liquid-like in nature (Figure 4A). In contrast, over time the condensates become more solid and are unable to fully fuse resulting in the formation of condensates with apparent substructure (Figure 4A). With this observation, we sought to determine if the oligomeric state of ARF19 inside condensates showed an analogous time-dependent maturation. Unlike condensates measured after sixteen hours, we found that newly formed ARF19 condensates contain few, if any, higher-order oligomers (Figure 4B, C). This suggests that there may be a relationship between the apparent fluidity and the accumulation of higher order oligomers within individual condensates.

Apart from imparting information with respect to the quantity of different oligomeric species within condensates, N\&B analysis can also reveal information with respect to the spatial distribution of oligomers within a condensate. N\&B analysis has previously revealed the distribution of oligomers within ARF19 condensates, finding that the higher order oligomers tend to be towards the center of the condensates and the monomers and dimers can be found towards the periphery [22]. After we found that the abundance of lower order and higher order oligomers is dramatically different between the early and late ARF19 condensates, we sought to examine whether the spatial distribution of lower and higher order oligomers was different between the early and late condensates. We found that the distribution of oligomers in early condensates was similar to that seen in the later condensates (Figure 5A). Therefore, while the relative abundance of different oligomeric species differs significantly between the early and late ARF19 condensates (Figure 5B), the general pattern where higher order oligomers tend to exist towards the center of the condensates and the lower order oligomers towards the periphery appears to be consistent over time. 
A.

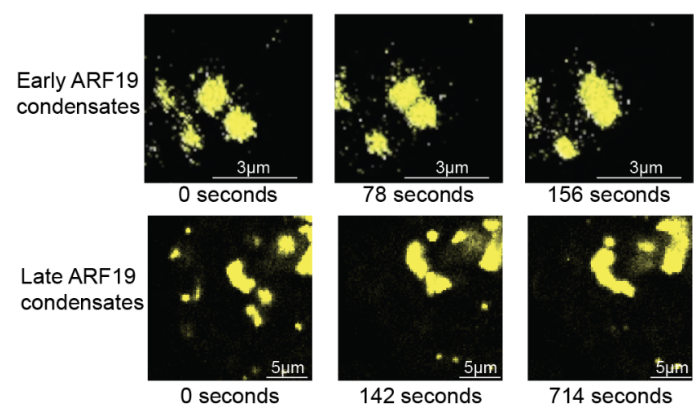

B.

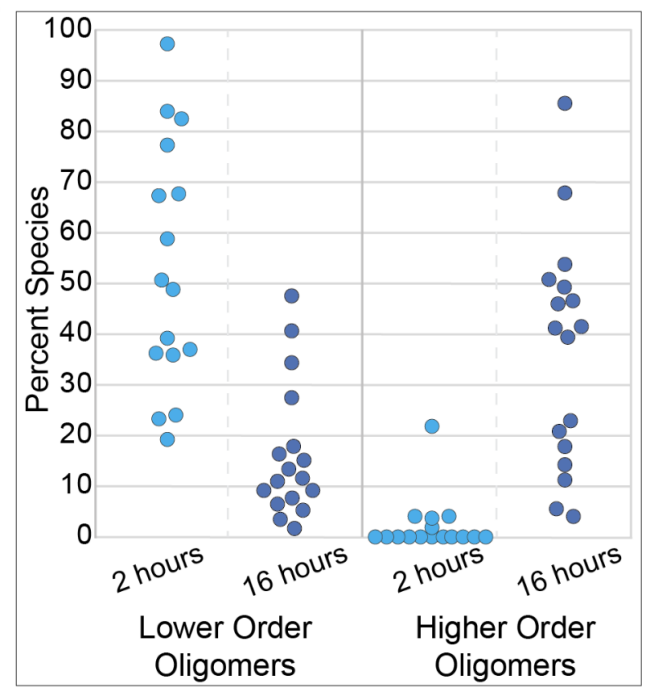

C.

\begin{tabular}{|l|c|c|}
\hline & 2 hours & 16 hours \\
\hline $\begin{array}{l}\text { Average \% } \\
\text { Lower Order Oligomers }\end{array}$ & 53.1 & 16.18 \\
\hline $\begin{array}{l}\text { Standard Deviation } \\
\text { Lower Order Oligomers }\end{array}$ & 24.23 & 13.35 \\
\hline $\begin{array}{l}\text { Average \% } \\
\text { Higher Order Oligomers }\end{array}$ & 2.18 & 36.28 \\
\hline $\begin{array}{l}\text { Standard Deviation } \\
\text { Higher Order Oligomers }\end{array}$ & 5.42 & 22.69 \\
\hline
\end{tabular}

Figure 4. ARF19 condensates are initially liquid-like and lack higher-order oligomers. (A) Time lapse images of wild-type ARF19 condensates in protoplasts. The top images are from the beginning of a time-lapse series started shortly after protoplast transfection (early ARF19 condensates) and show an example where the condensates have liquid-like behavior. The bottom images are from a later time point in a time lapse series (late ARF19 condensates) and show an example where the condensates appear to partially fuse but ultimately are unable to fully fuse resulting in a 'grape-bunch' like morphology. Note, time intervals between the bottom panels are not equal from panel to panel. (B) Each point shows the percent values for either lower order oligomers (left) or higher order oligomers (right) for individual ARF19 condensates approximately two or sixteen hours after protoplast transfection $\mathrm{N}=16$ ( 3 hours) and $\mathrm{N}=17$ (16 hours). (C) Table summarizing the data from figure panel $B$. 
A.

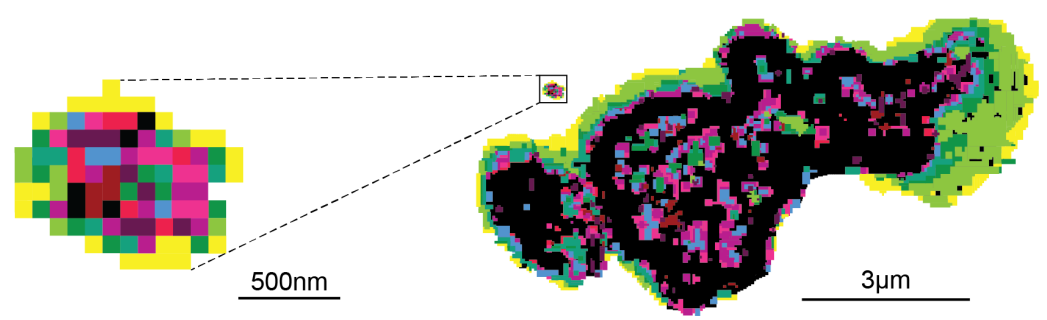

3 Hours 16 hours

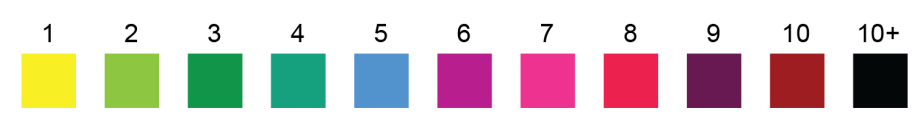

B.

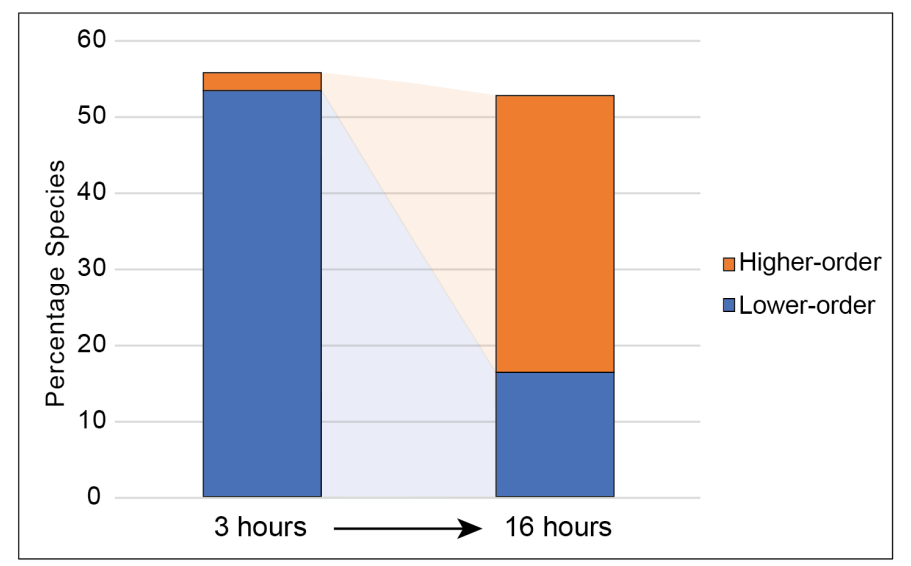

Figure 5. Distribution of oligomeric species in ARF19 condensates over space and time. (A) N\&B analysis showing the spatial distribution of various oligomeric species in early (left) and late (right) ARF19 condensates. Each oligomeric species corresponds to a different color. The size of the early condensate relative to the late condensate can be seen in the box towards the top left of the late condensate. (B) N\&B analysis showing the average percentage of higher-order and lower-order oligomers in ARF19 condensates in early condensates (left) and late condensates (right).

\section{Examining the relationship between oligomeric state and fluorescence recovery of individual condensates}

Finally, we examined the relationship between condensate fluidity and oligomeric state. During data acquisition we carried out N\&B followed by FRAP measurements on the same individual condensates, allowing us to directly correlate oligomeric state with condensate dynamics on a per-condensate basis. We found that the condensates formed by wild-type ARF19 showed a weak but positive correlation between the percent of lower order oligomers or of higher order oligomers and the percent recovery of individual condensates (Figure 6A, B). In contrast, the QtoS and QtoG variants showed very little correlation between either the percent of lower or higher order oligomers and fluorescence recovery for individual condensates. (Figures 6C-F). 
A.

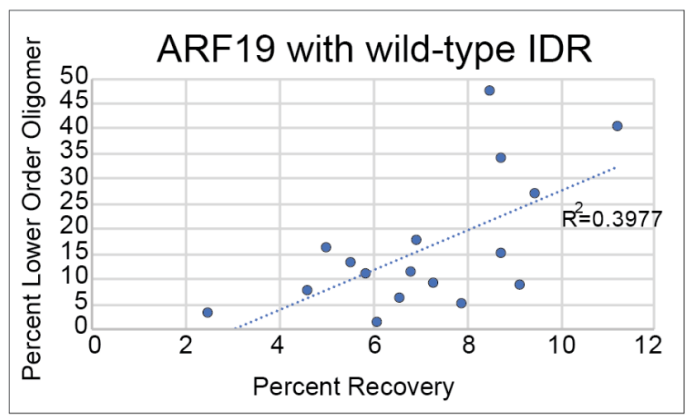

C.

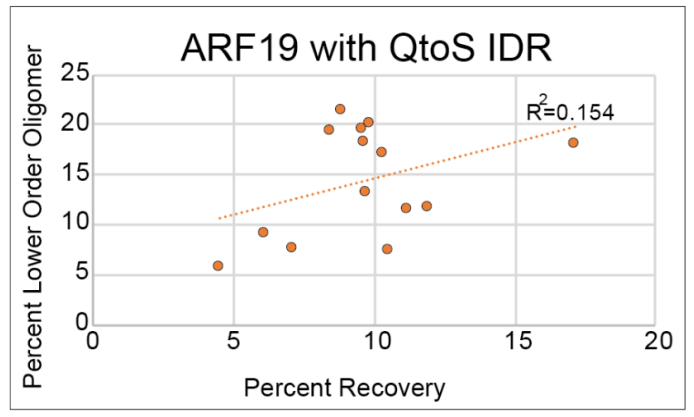

E.

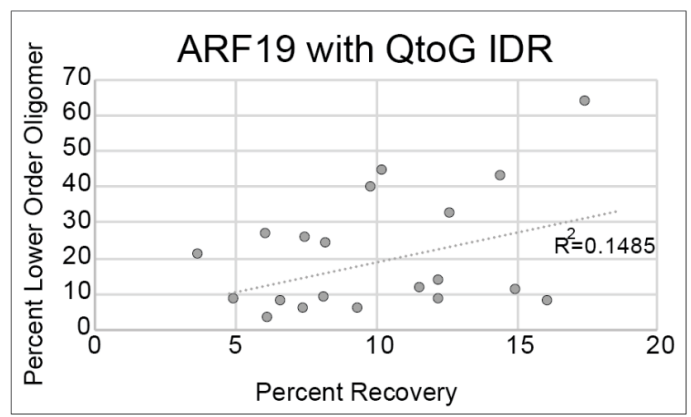

B.

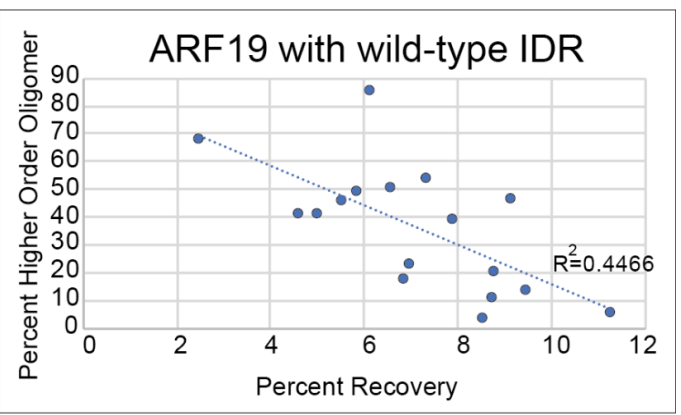

D.

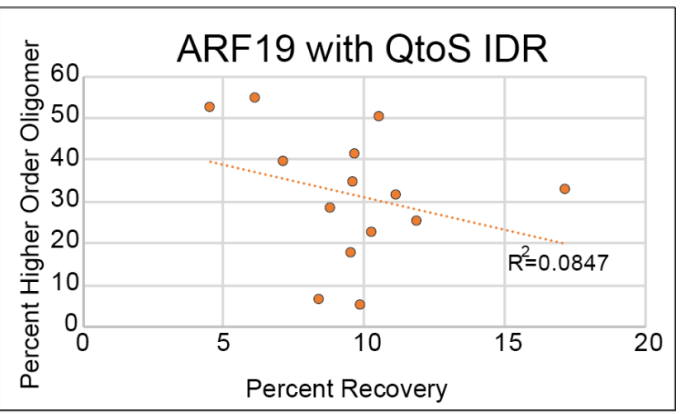

F.

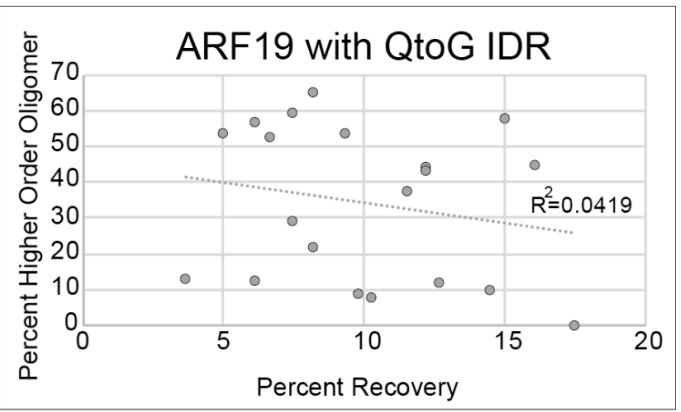

G.

\begin{tabular}{|c|c|c|}
\hline & Percent Lower Order Oligomer vs. Percent Recovery & Percent Higher Order Oligomer vs. Percent Recovery \\
\hline wild-type & $y=3.960 x-12.038$ & $y=-7.1311 x+87.096$ \\
\hline QtoS & $y=0.726 x+7.396$ & $y=-1.545 x+46.477$ \\
\hline QtoG & $y=1.646 x+4.308$ & $y=-1.133 x+45.348$ \\
\hline
\end{tabular}

Figure 6. IDR composition-dependent relationship between condensate oligomeric state and fluidity. (A-F) Each point shows N\&B and FRAP data for an individual condensate. Panels on the left show the relationship between the percent of lower order oligomers and the fluorescence recovery two minutes post-photobleaching for individual condensates whereas panels on the right show the relationship between higher order oligomers and percent recovery for individual condensates. Dashed lines are linear fit lines. $R^{2}$ values are shown near the linear fit lines. $N=17$ (WT), N=14 (QtoS), and $N=20$ (QtoG). (G) Equations for linear fit lines shown for each graph. 


\section{Discussion}

Biomolecular condensates have emerged as key organizers of cellular matter, whereby multivalent interactions underlie the assembly, recruitment, and regulation of a wide array of cellular bodies. The perspective that condensate formation is driven by intrinsically disordered regions has given way to a broader appreciation for distributed multivalency, whereby both disordered regions and folded oligomerization domains can make key contributions to the assembly of condensates [9]. Furthermore, condensate material state is increasingly being recognized as an important contributor to cellular function, either directly or indirectly [38-40].

In this study, we utilized ARF19 as a system to examine the role that IDR composition has on the emergent material properties of ARF19 condensates. Importantly, because of the inherent sensitivity that IDRs and more generally phase separation have to the surrounding solution environment, we carried out this work inside of protoplasts, which are plant cells that lack an external cell wall $[11,21]$. This allowed us to examine the effect that IDR composition has on condensate material properties within the cellular environment. In the context of material properties, this is of particular importance as there are known examples where condensates that exhibit liquid-like dynamics in vivo form non-dynamic, solid assemblies in vitro, suggesting that the solution environment has a direct influence on the material properties of condensates $[20,44]$. By first quantifying oligomeric state using Number and Brightness (N\&B) analysis and then examining fluidity using Fluorescence Recovery After Photobleaching (FRAP) for ARF19 variants with differing IDR compositions, we were able to assess the relationship between IDR composition and the apparent solidity and oligomeric state of ARF19 condensates. In addition, because we carried out these analyses sequentially for individual condensates, we were able to examine the relationship between the oligomeric state of the condensates and condensate fluidity, in an effective "single-condensate spectroscopy" type experiment.

Whereas the oligomerization domain of ARF19 promotes condensate formation through a well-defined binding interface, the mechanism by which the glutamine-rich IDR of ARF19 contributes is less obvious [22,71]. Extant work on polyglutamine has demonstrated its robust tendency to undergo self assembly, and previous studies have shown that the glutamine content in an IDR can impact the propensity of condensates to undergo time-dependent maturation and loss of dynamics [20,72-76]. In addition, prion-like domains, which are generally associated with aggregate or condensate formation, are frequently glutamine-rich [61,62,77-79]. In regards to the underlying mechanism by which glutamine contributes to material properties of condensates, glutamine-rich IDRs can form coiled-coils that have the capacity to facilitate protein-protein interactions and multimerization [76,79,80]. Therefore, in the context of ARF19, which has a glutamine-rich IDR with multiple polyglutamine stretches, the glutamine content may underlie the material properties of ARF19 condensates.

IDRs are frequently found to be both necessary and sufficient for biomolecular condensate formation. Despite their clear importance in condensate formation, little is known about the impact that IDR composition has on the emergent material properties of condensates in vivo. In addition, while multiple examples of proteins requiring both their IDR and 
oligomerization domain to form condensates have emerged, how IDR composition impacts condensate dynamics in this context is poorly understood. Here, through use of FRAP and Number and Brightness analysis (N\&B) coupled with altering the composition of the IDR in ARF19, we have begun to shed light on this question.

\section{IDR composition influences condensate dynamics}

From our FRAP analysis of condensates formed from wild-type ARF19 or our ARF19 variants, we found that the QtoS and QtoG variants resulted in condensates with slightly increased fluidity. While the IDR can tune condensate properties, it is worth noting that in all three cases ARF19 condensates were relatively solid at 16 hours post-transfection, reaching a maximum average fluorescence recovery after two minutes of $\sim 9.6 \%$ (QtoS and QtoG variants, Figure 2).

The behavior of the QtoG variant is in contrast to a previous study which found that changing glutamine to glycine in the FUS PLD resulted in the formation of more dynamic condensates that did not undergo time-dependent maturation [20]. There are numerous possible explanations for this discrepancy. First, the previous study assessed condensate fluidity in vitro whereas our QtoG variant was examined within the cellular environment. Secondly, FUS and ARF19 differ in more than just their IDRs, and multivalent interactions facilitated by other protein regions that differ between the two proteins may also contribute to the differing results. Finally, the composition and patterning of amino acids in the FUS PLD and the ARF19 PLD are substantially different, with the FUS PLD containing an abundance of tyrosine residues that are absent in the ARF19 PLD. As such, the differences between the two outcomes may simply be due to the different IDR compositions. Moreover, while both are glutamine rich, unlike the ARF19 PLD, the FUS PLD lacks contiguous glutamine tracts. Prior work has established that polyglycine shows poor solubility in water and exists in compact, collapsed conformations that can undergo self-assembly [81-84]. As such, the acquisition of polyglycine tracts in the QtoG variant may be an additional determinant that contributes to differences in assembly behavior compared to the FUS PLD variant.

Glutamine rich sequences have been shown to form coiled-coiled domains that drive oligomerization through a conditionally-structured interface $[80,85,86]$. Moreover, analysis of the ARF19 sequence with the COILS webserver predicts a coiled-coil domain in line with one of the glutamine-rich subregions in the IDR (Figure S1) [87]. However, we can largely exclude coiled-coils as a key determinant of protein oligomeric state given that glycine strongly impedes helix formation $[88,89]$. With this in mind, the QtoG variant should fundamentally prevent any possible coiled-coil association, yet no different in intra-condensate oligomeric state is observed across our three variants (Figure 3 ). While we cannot rule out the possibility that coiled-coil domains may influence condensate morphology, it is conceptually challenging to envisage a model in which the presence or absence of a coiled-coil domain does not influence oligomeric state yet alters higher-order assembly. As such, we interpret our results to mean the glutamine-rich IDR functions in a largely unstructured manner. 
The increased recovery dynamics observed in the QtoS variant may reflect various possible molecular origins. This result could report on a reduction in IDR-mediated interactions due to the replacement of a secondary amide sidechain (glutamine) for a hydroxyl group (serine). Alternatively it may reflect a change in residual structure, as implicated by work that suggests poly-serine might adopt a more expanded, rigid conformation [90]. Nevertheless, more work is needed to extrapolate results from simple homopolymeric peptides to observations in the context of full-length proteins.

ARF19 is part of a family of 23 ARFs in Arabidopsis that is broken into three separate clades [55]. The only ARFs currently known to form condensates in plants belong to clade $A$. These ARFs have characteristic glutamine-rich IDRs and are thought to act as transcriptional activators [91,92]. In contrast, there is currently no evidence that clade B ARFs form condensates in plants. Clade B ARFs are thought to be transcriptional repressors and contain serine-rich IDRs [91,92]. Our initial decision to alter the ARF19 IDR to become serine rich was based on the observation that none of the clade B ARFs, which contain serine-rich IDRs, are known to form condensates in plants. However, the minimal differences in solidity seen in our QtoS variant when compared to wild type ARF19 may suggest that the serine-rich nature of the clade B ARFs is not the inherent reason why these ARFs do not form condensates in plants.

\section{Oligomeric state is insensitive to IDR composition}

In contrast to our FRAP data, we were unable to identify significant differences in the oligomeric state between any of the ARF19 variants. We interpret this to mean that while the IDR has the capacity to modulate the material properties of ARF19 condensates, the oligomeric state may be less impacted or all together independent of IDR composition. However, we cannot unambiguously conclude this due to the limited number of variants we analyzed, and the resulting limited statistical power.

Given that in planta both the IDR and the PB1 oligomerization domain are necessary for condensate formation, it is almost certain that the IDR contributes to the multivalent interactions that are essential for condensate formation. In support of this notion, when we expressed the PB1 domain of ARF19 alone in protoplasts, even among the protoplasts with the highest apparent accumulation of the protein, we did not observe any condensates, nor did we detect higher order oligomers through our N\&B analysis (Table S1). In contrast, the PB1 domain forms multimers in vitro even in the absence of the IDR [71]. As such, our results support a model in which IDRs enhance the driving force for lower-order oligomers in vivo, but that this effect is sufficiently subtle that the QtoS and QtoG variants do not significantly perturb the effect vis-à-vis wild type. These results do not exclude the possibility that IDRs also stabilize higher order oligomers, a behavior we would expect to hold true. Future studies examining the oligomeric state of protein variants where IDRs are more dramatically altered, ideally in a way that minimizes the likelihood of the IDR contributing to multivalent interactions, should shed light on this question. 


\section{Oligomeric populations change during condensate maturation in living cells}

Given the observation that many condensates undergo time-dependent changes in dynamics and organization, we applied N\&B analysis to assess how the oligomeric state of molecules inside condensates changes as a function of time. While condensates measured immediately after assembly were composed predominantly of monomers, after 16 hours we found a much larger population of higher order oligomers. These results are consistent with a model in which the high local concentration of molecules within a condensate drives concentration-dependent higher-order assembly, which in turn offers a structural explanation for changes in condensate dynamics. As larger oligomers form, their internal re-arrangement will become increasingly constrained due to molecular entanglement such that a jamming transition may ultimately occur, leading to a kinetically arrested assembly.

While our results here are readily interpretable in the context of oligomerization driven by the PB1 domain, the same principle is applicable to other systems in which distinct modes of assembly, such as the assembly of liquid-like condensates driven by distributed aromatic motifs, followed by a liquid-to-solid transition driven by the acquisition of structured cross-beta interactions [46,93-97]. Our previous work demonstrated that oligomeric state in condensates varies as a function of spatial position across the condensate, with more lower-order species on the surface and higher-order species in the interior [22]. Taken together, N\&B analysis reveals that, at least for ARF19, oligomeric state varies in both space and time, revealing a rich and perhaps surprisingly complex oligomeric landscape of intra-condensate molecules.

\section{Oligomeric state can influence condensate dynamics}

By carrying out N\&B analysis followed by FRAP on individual condensates, we were able to establish a weak but clear correlation between the oligomeric state and the fluidity of condensates. This relationship is consistent with our finding that liquid-like ARF19 condensates examined shortly after formation do not contain substantial accumulations of higher order oligomers.

In contrast to wild-type ARF19 condensates, we did not observe a strong correlation between oligomeric state and condensate dynamics for condensates formed by either the QtoS or QtoG ARF19 variants. While it is possible that altering the IDR composition disrupted this relationship in some non-obvious way, it is also possible that the N\&B data simply had too much noise for the QtoS and QtoG variants for us to see a clear relationship in this instance. Studying the behavior of biomolecular condensates in vivo is extremely challenging due to the inherent dynamic nature of the intracellular environment. In anecdotal support of this, in our protoplast system the ARF19 (or ARF19 variant) condensates were frequently highly mobile making capturing high-quality microscopy data challenging. Approximately $95 \%$ of the data acquired for this study had to be discarded prior to analysis simply due to the condensate moving out of view during the $\sim 4$ minutes of data acquisition. Furthermore, given the substantially reduced size of condensates formed by the QtoS and QtoG variants, the likelihood of the variant condensates moving out of the Z-plane during acquisition was much higher than for wild-type condensates. This is not to say that in vivo studies of this type should not be attempted; rather, results such as 
the discrepancy between the presence of a relationship between the oligomeric state and the apparent fluidity of condensates formed by wild-type ARF19 and the lack of such a relationship in condensates formed by the QtoS and QtoG should be carefully considered. However, the prospect of ever-improving technologies that allow for more rapid and accurate acquisition of in vivo data will inevitably allow for a more accurate assessment of this relationship.

\section{Conclusions}

In all, our work here offers direct insight into the relationships between IDR composition, condensate dynamics, and oligomeric state for a condensate-forming protein containing both an IDR and an oligomerization domain. Our results support an emerging consensus in which IDR composition impacts the emergent physical properties of biomolecular condensates both in vitro and in vivo. In contrast, our work suggests that, at least in the ARF19 system, IDR composition has a more limited role in governing the oligomeric state of in vivo condensates. Taken together, these results support a general model in which structurally and chemically orthogonal multivalent interactions can contribute distinct attributes to the emergent properties of biomolecular condensates. 


\section{Competing interests}

A.S.H. is a scientific consultant with Dewpoint Therapeutics. R.J.E. and L.C.S. declare no competing interests.

\section{Funding}

This research was funded by the William H. Danforth Plant Science Fellowship (to R.J.E.), the National Science Foundation (IOS-1453650 to L.C.S.), the National Institutes of Health (R35GM136338 to L.C.S.), and start-up funds from Washington University in St. Louis (to A.S.H.).

\section{Author's contributions}

R.J.E., A.S.H., and L.C.S. designed this study and interpreted data. R.J.E. performed all described experiments. All authors read and approved the final manuscript.

\section{Acknowledgements}

We thank Ishan Taneja for helpful comments on this manuscript. 


\section{References}

1. Cohan MC, Pappu RV. Making the Case for Disordered Proteins and Biomolecular Condensates in Bacteria. Trends Biochem Sci. 2020;45:668-80.

2. Emenecker RJ, Holehouse AS, Strader LC. Emerging Roles for Phase Separation in Plants. Dev Cell. 2020;55:69-83.

3. Banani SF, Lee HO, Hyman AA, Rosen MK. Biomolecular condensates: organizers of cellular biochemistry. Nat Rev Mol Cell Biol. 2017;18:285-98.

4. Brangwynne CP, Eckmann CR, Courson DS, Rybarska A, Hoege C, Gharakhani J, et al. Germline $\mathrm{P}$ granules are liquid droplets that localize by controlled dissolution/condensation. Science. 2009;324:1729-32.

5. Boeynaems S, Alberti S, Fawzi NL, Mittag T, Polymenidou M, Rousseau F, et al. Protein Phase Separation: A New Phase in Cell Biology. Trends Cell Biol. 2018;28:420-35.

6. Hyman AA, Weber CA, Jülicher F. Liquid-liquid phase separation in biology. Annu Rev Cell Dev Biol. 2014;30:39-58.

7. Brangwynne CP, Tompa P, Pappu RV. Polymer physics of intracellular phase transitions. Nat Phys. 2015;11:899-904.

8. Li P, Banjade S, Cheng H-C, Kim S, Chen B, Guo L, et al. Phase transitions in the assembly of multivalent signalling proteins. Nature. 2012;483:336-40.

9. Choi J-M, Holehouse AS, Pappu RV. Physical Principles Underlying the Complex Biology of Intracellular Phase Transitions. Annu Rev Biophys. 2020;49:107-33.

10. Sanders DW, Kedersha N, Lee DSW, Strom AR, Drake V, Riback JA, et al. Competing Protein-RNA Interaction Networks Control Multiphase Intracellular Organization. Cell. Elsevier; 2020;181:306-24.e28.

11. Holehouse AS. Chapter 7 - IDPs and IDRs in biomolecular condensates. In: Salvi N, editor. Intrinsically Disordered Proteins. Academic Press; 2019. p. 209-55.

12. Lin Y, Protter DSW, Rosen MK, Parker R. Formation and maturation of phase-separated liquid droplets by RNA-binding proteins. Mol Cell. 2015;60:208-19.

13. Han TW, Kato M, Xie S, Wu LC, Mirzaei H, Pei J, et al. Cell-free formation of RNA granules: bound RNAs identify features and components of cellular assemblies. Cell. Elsevier; 2012;149:768-79.

14. Kato M, Han TW, Xie S, Shi K, Du X, Wu LC, et al. Cell-free formation of RNA granules: low complexity sequence domains form dynamic fibers within hydrogels. Cell. 2012;149:753-67.

15. Wright PE, Dyson HJ. Intrinsically unstructured proteins: re-assessing the protein structure-function paradigm. J Mol Biol. 1999;293:321-31.

16. van der Lee R, Buljan M, Lang B, Weatheritt RJ, Daughdrill GW, Dunker AK, et al. Classification of intrinsically disordered regions and proteins. Chem Rev. 2014;114:6589-631. 17. Das RK, Ruff KM, Pappu RV. Relating sequence encoded information to form and function of intrinsically disordered proteins. Curr Opin Struct Biol. 2015;32:102-12.

18. Elbaum-Garfinkle S, Kim Y, Szczepaniak K, Chen CC-H, Eckmann CR, Myong S, et al. The 
disordered $\mathrm{P}$ granule protein LAF-1 drives phase separation into droplets with tunable viscosity and dynamics. Proc Natl Acad Sci U S A. 2015;112:7189-94.

19. Nott TJ, Petsalaki E, Farber P, Jervis D, Fussner E, Plochowietz A, et al. Phase transition of a disordered nuage protein generates environmentally responsive membraneless organelles. Mol Cell. 2015;57:936-47.

20. Wang J, Choi J-M, Holehouse AS, Lee HO, Zhang X, Jahnel M, et al. A Molecular Grammar Governing the Driving Forces for Phase Separation of Prion-like RNA Binding Proteins. Cell. 2018;174:688-99.e16.

21. Martin EW, Holehouse AS. Intrinsically disordered protein regions and phase separation: sequence determinants of assembly or lack thereof. Emerg Top Life Sci. 2020;4:307-29.

22. Powers SK, Holehouse AS, Korasick DA, Schreiber KH, Clark NM, Jing $H$, et al.

Nucleo-cytoplasmic Partitioning of ARF Proteins Controls Auxin Responses in Arabidopsis thaliana. Mol Cell. 2019;76:177-90.e5.

23. Yang P, Mathieu C, Kolaitis R-M, Zhang P, Messing J, Yurtsever U, et al. G3BP1 Is a Tunable Switch that Triggers Phase Separation to Assemble Stress Granules. Cell. 2020;181:325-45.e28.

24. Guillén-Boixet J, Kopach A, Holehouse AS, Wittmann S, Jahnel M, Schlüßler R, et al. RNA-Induced Conformational Switching and Clustering of G3BP Drive Stress Granule Assembly by Condensation. Cell. 2020;181:346-61.e17.

25. Marzahn MR, Marada S, Lee J, Nourse A, Kenrick S, Zhao H, et al. Higher-order oligomerization promotes localization of SPOP to liquid nuclear speckles. EMBO J. 2016;35:1254-75.

26. Bouchard JJ, Otero JH, Scott DC, Szulc E, Martin EW, Sabri N, et al. Cancer Mutations of the Tumor Suppressor SPOP Disrupt the Formation of Active, Phase-Separated Compartments. Mol Cell. 2018;72:19-36.e8.

27. Riback JA, Katanski CD, Kear-Scott JL, Pilipenko EV, Rojek AE, Sosnick TR, et al. Stress-Triggered Phase Separation Is an Adaptive, Evolutionarily Tuned Response. Cell. 2017;168:1028-40.e19.

28. Taratuta VG, Holschbach A, Thurston GM, Blankschtein D, Benedek GB. Liquid-liquid phase separation of aqueous lysozyme solutions: effects of $\mathrm{pH}$ and salt identity. J Phys Chem.

American Chemical Society; 1990;94:2140-4.

29. Wang A, Conicella AE, Schmidt HB, Martin EW, Rhoads SN, Reeb AN, et al. A single $\mathrm{N}$-terminal phosphomimic disrupts TDP-43 polymerization, phase separation, and RNA splicing. EMBO J [Internet]. 2018;37. Available from: http://dx.doi.org/10.15252/embj.201797452 30. Shin Y, Berry J, Pannucci N, Haataja MP, Toettcher JE, Brangwynne CP. Spatiotemporal Control of Intracellular Phase Transitions Using Light-Activated optoDroplets. Cell.

2017;168:159-71.e14.

31. Dao TP, Martyniak B, Canning AJ, Lei Y, Colicino EG, Cosgrove MS, et al. ALS-Linked Mutations Affect UBQLN2 Oligomerization and Phase Separation in a Position- and Amino Acid-Dependent Manner. Structure. Elsevier; 2019;27:937-51.e5.

32. Larson AG, Elnatan D, Keenen MM, Trnka MJ, Johnston JB, Burlingame AL, et al. Liquid 
droplet formation by HP1 $\alpha$ suggests a role for phase separation in heterochromatin. Nature. nature.com; 2017;547:236-40.

33. Strom AR, Emelyanov AV, Mir M, Fyodorov DV, Darzacq X, Karpen GH. Phase separation drives heterochromatin domain formation. Nature. 2017;547:241-5.

34. Cubuk J, Alston JJ, Jeremías Incicco J, Singh S, Stuchell-Brereton MD, Ward MD, et al. The SARS-CoV-2 nucleocapsid protein is dynamic, disordered, and phase separates with RNA. Nat Commun. Nature Publishing Group; 2021;12:1-17.

35. Holehouse AS, Pappu RV. Functional Implications of Intracellular Phase Transitions.

Biochemistry. 2018;57:2415-23.

36. Franzmann TM, Alberti S. Prion-like low-complexity sequences: Key regulators of protein solubility and phase behavior. J Biol Chem. 2019;294:7128-36.

37. Franzmann TM, Jahnel M, Pozniakovsky A, Mahamid J, Holehouse AS, Nüske E, et al. Phase separation of a yeast prion protein promotes cellular fitness. Science [Internet]. 2018;359. Available from: http://dx.doi.org/10.1126/science.aao5654

38. Lasker K, Boeynaems S, Lam V, Stainton E, Jacquemyn M, Daelemans D, et al. A modular platform for engineering function of natural and synthetic biomolecular condensates [Internet]. Cold Spring Harbor Laboratory. 2021 [cited 2021 Feb 4]. p. 2021.02.03.429226. Available from: https://www.biorxiv.org/content/10.1101/2021.02.03.429226v1

39. Sankaranarayanan M, Emenecker RJ, Jahnel M, Irmela R E, Wayland MT, Alberti S, et al. The arrested state of processing bodies supports mRNA regulation in early development [Internet]. Cold Spring Harbor Laboratory. 2021 [cited 2021 Mar 17]. p. 2021.03.16.435709. Available from: https://www.biorxiv.org/content/10.1101/2021.03.16.435709v1

40. Weber SC. Sequence-encoded material properties dictate the structure and function of nuclear bodies. Curr Opin Cell Biol. 2017;46:62-71.

41. Jawerth L, Fischer-Friedrich E, Saha S, Wang J, Franzmann T, Zhang X, et al. Protein condensates as aging Maxwell fluids. Science. American Association for the Advancement of Science; 2020;370:1317-23.

42. Woodruff JB, Hyman AA, Boke E. Organization and Function of Non-dynamic Biomolecular Condensates. Trends Biochem Sci. 2018;43:81-94.

43. Boke E, Ruer M, Wühr M, Coughlin M, Lemaitre R, Gygi SP, et al. Amyloid-like Self-Assembly of a Cellular Compartment. Cell. 2016;166:637-50.

44. Patel A, Lee HO, Jawerth L, Maharana S, Jahnel M, Hein MY, et al. A Liquid-to-Solid Phase Transition of the ALS Protein FUS Accelerated by Disease Mutation. Cell. 2015;162:1066-77. 45. Posey AE, Holehouse AS, Pappu RV. Phase Separation of Intrinsically Disordered Proteins. Methods Enzymol. 2018;611:1-30.

46. Schmidt HB, Barreau A, Rohatgi R. Phase separation-deficient TDP43 remains functional in splicing. Nat Commun. 2019;10:4890.

47. Schuster BS, Dignon GL, Tang WS, Kelley FM, Ranganath AK, Jahnke CN, et al. Identifying sequence perturbations to an intrinsically disordered protein that determine its phase-separation behavior. Proc Natl Acad Sci U S A [Internet]. 2020; Available from:

http://dx.doi.org/10.1073/pnas.2000223117 
48. Martin EW, Holehouse AS, Peran I, Farag M, Jeremias Incicco J, Bremer A, et al. Valence and patterning of aromatic residues determine the phase behavior of prion-like domains [Internet]. Science. 2020. p. 694-9. Available from: http://dx.doi.org/10.1126/science.aaw8653 49. Cuevas-Velazquez CL, Vellosillo T, Guadalupe K, Broder Schmidt H, Yu F, Moses D, et al. Intrinsically disordered protein biosensor tracks the physical-chemical effects of osmotic stress on cells [Internet]. Cold Spring Harbor Laboratory. 2021 [cited 2021 Feb 18]. p.

2021.02.17.431712. Available from:

https://www.biorxiv.org/content/10.1101/2021.02.17.431712v1.article-info

50. Moses D, Yu F, Ginell GM, Shamoon NM, Koenig PS, Holehouse AS, et al. Revealing the Hidden Sensitivity of Intrinsically Disordered Proteins to their Chemical Environment. J Phys Chem Lett. 2020;11:10131-6.

51. Holehouse AS, Sukenik S. Controlling Structural Bias in Intrinsically Disordered Proteins Using Solution Space Scanning. J Chem Theory Comput. 2020;16:1794-805.

52. Wicky BIM, Shammas SL, Clarke J. Affinity of IDPs to their targets is modulated by ion-specific changes in kinetics and residual structure. Proc Natl Acad Sci U S A.

2017;114:9882-7.

53. Shammas SL, Crabtree MD, Dahal L, Wicky BIM, Clarke J. Insights into coupled folding and binding mechanisms from kinetic studies. J Biol Chem. ASBMB; 2016;291:6689-95.

54. Rogers JM, Wong CT, Clarke J. Coupled folding and binding of the disordered protein PUMA does not require particular residual structure. J Am Chem Soc. 2014;136:5197-200.

55. Powers SK, Strader LC. Regulation of auxin transcriptional responses. Dev Dyn. 2020;249:483-95.

56. Last RL, Fink GR. Tryptophan-Requiring Mutants of the Plant Arabidopsis thaliana. Science. 1988;240:305-10.

57. Haughn GW, Somerville C. Sulfonylurea-resistant mutants of Arabidopsis thaliana. Mol Gen Genet. 1986;204:430-4.

58. Wu F-H, Shen S-C, Lee L-Y, Lee S-H, Chan M-T, Lin C-S. Tape-Arabidopsis Sandwich - a simpler Arabidopsis protoplast isolation method. Plant Methods. 2009;5:16.

59. Digman MA, Brown CM, Sengupta P, Wiseman PW. Measuring fast dynamics in solutions and cells with a laser scanning microscope. Biophys J [Internet]. Elsevier; 2005; Available from: https://www.sciencedirect.com/science/article/pii/S000634950572779X

60. Schindelin J, Arganda-Carreras I, Frise E, Kaynig V, Longair M, Pietzsch T, et al. Fiji: an open-source platform for biological-image analysis. Nat Methods. 2012;9:676-82.

61. Lancaster AK, Nutter-Upham A, Lindquist S, King OD. PLAAC: a web and command-line application to identify proteins with prion-like amino acid composition. Bioinformatics.

2014;30:2501-2.

62. Alberti S, Halfmann R, King O, Kapila A, Lindquist S. A systematic survey identifies prions and illuminates sequence features of prionogenic proteins. Cell. Elsevier Ltd; 2009;137:146-58. 63. Audas TE, Audas DE, Jacob MD, Ho JJD, Khacho M, Wang M, et al. Adaptation to Stressors by Systemic Protein Amyloidogenesis. Dev Cell. 2016;39:155-68.

64. Hubstenberger A, Noble SL, Cameron C, Evans TC. Translation repressors, an RNA 
helicase, and developmental cues control RNP phase transitions during early development. Dev Cell. Elsevier; 2013;27:161-73.

65. Woodruff JB, Wueseke O, Viscardi V, Mahamid J, Ochoa SD, Bunkenborg J, et al. Centrosomes. Regulated assembly of a supramolecular centrosome scaffold in vitro. Science. 2015;348:808-12.

66. Arnon ZA, Vitalis A, Levin A, Michaels TCT, Caflisch A, Knowles TPJ, et al. Dynamic microfluidic control of supramolecular peptide self-assembly. Nat Commun. 2016;7:13190. 67. Boeynaems S, Holehouse AS, Weinhardt V, Kovacs D, Van Lindt J, Larabell C, et al. Spontaneous driving forces give rise to protein-RNA condensates with coexisting phases and complex material properties. Proc Natl Acad Sci U S A. 2019;116:7889-98.

68. Digman MA, Dalal R, Horwitz AF, Gratton E. Mapping the number of molecules and brightness in the laser scanning microscope. Biophys J. 2008;94:2320-32.

69. Linsenmeier M, Hondele M, Grigolato F, Secchi E, Weis K, Arosio P. Dynamic arrest and aging of biomolecular condensates are regulated by low-complexity domains, RNA and biochemical activity [Internet]. bioRxiv. 2021 [cited 2021 Apr 13]. p. 2021.02.26.433003. Available from: https://www.biorxiv.org/content/10.1101/2021.02.26.433003v1.abstract 70. Molliex A, Temirov J, Lee J, Coughlin M, Kanagaraj AP, Kim HJ, et al. Phase Separation by Low Complexity Domains Promotes Stress Granule Assembly and Drives Pathological Fibrillization. Cell. 2015;163:123-33.

71. Korasick DA, Westfall CS, Lee SG, Nanao MH, Dumas R, Hagen G, et al. Molecular basis for AUXIN RESPONSE FACTOR protein interaction and the control of auxin response repression. Proc Natl Acad Sci U S A. 2014;111:5427-32.

72. Pappu RV, Wang X, Vitalis A, Crick SL. A polymer physics perspective on driving forces and mechanisms for protein aggregation - Highlight Issue: Protein Folding. Arch Biochem Biophys. 2008;469:132-41.

73. Posey AE, Ruff KM, Harmon TS, Crick SL, Li A, Diamond MI, et al. Profilin reduces aggregation and phase separation of huntingtin $\mathrm{N}$-terminal fragments by preferentially binding to soluble monomers and oligomers. J Biol Chem. 2018;293:3734-46.

74. Crick SL, Jayaraman M, Frieden C, Wetzel R, Pappu RV. Fluorescence correlation spectroscopy shows that monomeric polyglutamine molecules form collapsed structures in aqueous solutions. Proc Natl Acad Sci U S A. 2006;103:16764-9.

75. Crick SL, Ruff KM, Garai K, Frieden C, Pappu RV. Unmasking the roles of N- and C-terminal flanking sequences from exon 1 of huntingtin as modulators of polyglutamine aggregation. Proc Natl Acad Sci U S A. 2013;110:20075-80.

76. Peskett TR, Rau F, O'Driscoll J, Patani R, Lowe AR, Saibil HR. A Liquid to Solid Phase Transition Underlying Pathological Huntingtin Exon1 Aggregation. Mol Cell. 2018;70:588-601.e6.

77. Du Z. The complexity and implications of yeast prion domains. Prion. 2011;5:311-6.

78. King OD, Gitler AD, Shorter J. The tip of the iceberg: RNA-binding proteins with prion-like domains in neurodegenerative disease. Brain Res. 2012;1462:61-80.

79. Murthy AC, Dignon GL, Kan Y, Zerze GH, Parekh SH, Mittal J, et al. Molecular interactions 
underlying liquid-liquid phase separation of the FUS low-complexity domain. Nat Struct Mol Biol. Nature Publishing Group; 2019;26:637-48.

80. Fiumara F, Fioriti L, Kandel ER, Hendrickson WA. Essential role of coiled coils for aggregation and activity of $\mathrm{Q} / \mathrm{N}$-rich prions and $\mathrm{Poly} Q$ proteins. Cell. 2010;143:1121-35.

81. Teufel DP, Johnson CM, Lum JK, Neuweiler H. Backbone-driven collapse in unfolded protein chains. J Mol Biol. 2011;409:250-62.

82. Karandur D, Harris RC, Pettitt BM. Protein collapse driven against solvation free energy without H-bonds: Protein Collapse Against Solvation Free Energy. Protein Sci. Wiley; 2016;25:103-10.

83. Asthagiri D, Karandur D, Tomar DS, Pettitt BM. Intramolecular Interactions Overcome Hydration to Drive the Collapse Transition of Gly15. J Phys Chem B. 2017;121:8078-84. 84. Holehouse AS, Garai K, Lyle N, Vitalis A, Pappu RV. Quantitative assessments of the distinct contributions of polypeptide backbone amides versus side chain groups to chain expansion via chemical denaturation. J Am Chem Soc. 2015;137:2984-95.

85. O'Shea EK, Lumb KJ, Kim PS. Peptide "Velcro": design of a heterodimeric coiled coil. Curr Biol. Elsevier; 1993;3:658-67.

86. Eckert DM, Malashkevich VN, Kim PS. Crystal structure of GCN4-pIQI, a trimeric coiled coil with buried polar residues. J Mol Biol. Elsevier; 1998;284:859-65.

87. Lupas A, Van Dyke M, Stock J. Predicting coiled coils from protein sequences. Science. JSTOR; 1991;252:1162-4.

88. Chou PY, Fasman GD. Prediction of the secondary structure of proteins from their amino acid sequence. Adv Enzymol Relat Areas Mol Biol. 1978;47:45-148.

89. Imai K, Mitaku S. Mechanisms of secondary structure breakers in soluble proteins.

Biophysics . 2005;1:55-65.

90. van Rosmalen M, Krom M, Merkx M. Tuning the Flexibility of Glycine-Serine Linkers To Allow Rational Design of Multidomain Proteins. Biochemistry. 2017;56:6565-74.

91. Guilfoyle TJ, Hagen G. Auxin response factors. J Plant Growth Regul [Internet]. Springer; 2001; Available from: https://link.springer.com/content/pdf/10.1007/s003440010026.pdf

92. Mutte SK, Kato H, Rothfels C, Melkonian M, Wong GK-S, Weijers D. Origin and evolution of the nuclear auxin response system. Elife [Internet]. elifesciences.org; 2018;7. Available from: http://dx.doi.org/10.7554/eLife.33399

93. Guenther EL, Cao Q, Trinh H, Lu J, Sawaya MR, Cascio D, et al. Atomic structures of TDP-43 LCD segments and insights into reversible or pathogenic aggregation. Nat Struct Mol Biol. Springer US; 2018;25:463-71.

94. Conicella AE, Dignon GL, Zerze GH, Schmidt HB, D’Ordine AM, Kim YC, et al. TDP-43 a-helical structure tunes liquid-liquid phase separation and function. Proc Natl Acad Sci U S A. National Academy of Sciences; 2020;117:5883-94.

95. Conicella AE, Zerze GH, Mittal J, Fawzi NL. ALS Mutations Disrupt Phase Separation Mediated by $\alpha$-Helical Structure in the TDP-43 Low-Complexity C-Terminal Domain. Structure [Internet]. 2016; Available from: http://dx.doi.org/10.1016/j.str.2016.07.007

96. Cao Q, Boyer DR, Sawaya MR, Ge P, Eisenberg DS. Cryo-EM structures of four 
bioRxiv preprint doi: https://doi.org/10.1101/2021.04.18.440340; this version posted April 19, 2021. The copyright holder for this preprint (which was not certified by peer review) is the author/funder. All rights reserved. No reuse allowed without permission.

polymorphic TDP-43 amyloid cores. Nat Struct Mol Biol. nature.com; 2019;26:619-27. 97. Bolognesi B, Faure AJ, Seuma M, Schmiedel JM, Tartaglia GG, Lehner B. The mutational landscape of a prion-like domain. Nat Commun. 2019;10:4162. 